\title{
ON THE LIMIT FUNCTIONS OF SEQUENCES OF CONTINUOUS FUNCTIONS CONVERGING RELATIVELY UNIFORMLY*
}

\section{E. W. CHITTENDEN}

1. The present paper is a continuation of a previous paper on the subject of relatively uniform convergence of sequences of functions of a single real variable. $\dagger$ The results of both papers admit of immediate extension to more general domains. A sequence $\left\{f_{n}(x)\right\}$ of functions, defined on an interval $I \equiv(a \leqq x \leqq b)$, converges relatively uniformly to a limit function $f(x)$ if there exists a function $\sigma(x)$, called a scale function $\sigma(x)$ such that for every small positive number $e$ there is an integer $n_{e}$ such that for every $n$ greater than $n_{e}$ the inequality

$$
\left|f(x)-f_{n}(x)\right| \leqq e|\sigma(x)|
$$

holds uniformly in $x$ on the interval $I . \ddagger$

It was shown in the previous paper that a sequence may converge without converging relatively uniformly. In fact there exist sequences of continuous functions which converge to a continuous limit but not relatively uniformly. If the convergence is relatively uniform the interval $I$ is the sum of an enumerable infinity of sets on which the convergence is uniform. Conversely, if $I$ is the sum of an enumerable infinity of sets on each of which the convergence is uniform or even relatively uniform, then the convergence is relatively uniform on $I$. It will be observed that uniform convergence is the special case of relatively uniform convergence in which the scale function is a non-zero constant.

The present paper is devoted to the study of the limit functions of sequences of continuous functions converging relatively uniformly. It is found that if $f(x)$ is such a function, then the discontinuities of $f(x)$ relative to any perfect set $P$ are non-dense on $P$. Following methods due to Baire the condition is found to be sufficient. The result suggests a method of classification

\footnotetext{
* Presented to the Society, December 28, 1917.

$\dagger \mathrm{E}$. W. Chittenden, Relatively uniform convergence of sequences of functions. These Trans a c ti o n s, vol. 1.5 (1914), pp. 197-201.

$\ddagger$ The concept and definition of relatively uniform convergence are due to E. H. Moore. Cf. An Introduction to a Form of General Analysis, The New Haven Mathematical Colloquium (Yale University Press, New Haven, 1910), pp. 1-150.
} 
of functions which characterizes important subclasses of the classes defined by Baire.

The paper contains an example of a function $f(x)$ represented everywhere by its Fourier series, although that series is not relatively uniformly convergent in any interval.

2. Let $E$ be any subset of the interval $I$. The points of $E$ contained in any subinterval of $I$ form a portion* of $E$. If $E$ is closed (perfect) any portion of $E$ is closed (perfect, with the possible exception of the right and left hand end points):

LEMmA. If $\left\{f_{n}(x)\right\}$ is a sequence of continuous functions converging uniformly on an open set $Q$, the limit function $f(x)$ of the sequence is continuous, not only on $Q$, but also on $Q^{\circ}=Q+Q^{\prime}$.

Since the convergence is uniform on $Q$ we have for every point $x$ in $Q$

$$
\left|f_{n}(x)-f_{n+p}(x)\right| \leqq e
$$

where $n$ depends only on $e$ and $p$ is arbitrary. The same inequality holds on $Q^{\circ}$, since the functions $f_{n}(x)$ are continuous. Hence the sequence converges uniformly on $Q^{\circ}$, and $f(x)$ must be continuous on $Q^{\circ}$.

Let $f(x)$ be the limit function of a sequence $\left\{f_{n}(x)\right\}$ of continuous functions converging on a perfect set $E$ uniformly relative to a scale function $\sigma(x)$. Denote by $Q_{k}$ the set of all points of $E$ at which $\sigma(x)<k$. The convergence is uniform on $Q_{k}$. It follows from the lemma that $f(x)$ is continuous on $Q_{k}^{0}=Q_{k}+Q_{k}^{\prime}$. The discontinuities of $f(x)$ cannot be dense on $E$. For, if they are dense on $E$, then $Q_{k}^{0}$ can contain no portion of $E$, that is $Q_{k}^{0}$ must be a closed non-dense subset of $E$. But every point of $E$ is in some one of the sets $Q_{k}$. That is $E$ is the sum of an enumerable infinity of sets of the first category with respect to itself. As this is impossible, the proposition is proved. We have then:

THEOREM 1. If a sequence $\left\{f_{n}(x)\right\}$ of continuous functions converges relatively uniformly to a limit function $f(x)$, then the discontinuities of $f(x)$ relative to any perfect set are non-dense on that set.

3. Theorem 2. If $f(x)$ is defined on a perfect set $P$ contained in an interval $I$, and if the discontinuities of $f(x)$ relative to every perfect set $E$ contained in $P$ are non-dense on $E$, there exists a sequence $\left\{f_{n}(x)\right\}$ of functions, each continuous on $P$, converging to $f(x)$ on $P$ relatively uniformly.

The following proof of this theorem is based upon the proof by Baire of the corresponding theorem for pointwise discontinuous functions. $\dagger$

Let $P_{0}$ denote the perfect set on which $f(x)$ is defined. Denote by $P_{1}$ the set of points of discontinuity of $f(x)$ relative to $P_{0}$ together with their

* Cf. Baire, Leçons sur les fonctions discontinues, Paris (1905), Gauthier-Villars, p. 105.

$\dagger$ Cf. Baire, loc. cit., pp. 110-120. 
limit points. The set $P_{1}$ is closed and, by hypothesis, non-dense on $P_{0}$. Denote by $P_{1}^{\Omega}$ the perfect component of $P_{1}$. In general, if $\gamma$ denotes a transfinite ordinal of the first class, we shall denote by $P_{\gamma+1}$ the class of all points of discontinuity of $f(x)$ relative to $P_{\gamma}^{\Omega}$ together with their limit points. If $\gamma$ is of the second class, $P_{\gamma}$ is the class of all points common to the classes $P_{\gamma}^{\prime}\left(\gamma^{\prime}<\gamma\right)$. There is a least index $\beta$, such that $P_{\beta}$ is not null, while $P_{\beta+1}$ is a null class*. The class $P=P_{0}$ admits the representation

$$
P=\sum P_{\gamma}-P_{\gamma+1},
$$

the sum extending over all numbers $\alpha \leqq \beta$. Denoting by $P_{\gamma}^{\nu}$ the $\nu$ th derivative of $P_{\gamma}$, we have

$$
\begin{aligned}
P_{\gamma}-P_{\gamma+1} & =P_{\gamma}-P_{\gamma}^{\Omega}+P_{\gamma}^{\Omega}-P_{\gamma+1} \\
& =\sum P_{\gamma}^{\nu}-P_{\gamma}^{\nu+1}+P_{\gamma}^{\Omega}-P_{\gamma+1} \quad(\nu=0,1,2, \cdots<\Omega) .
\end{aligned}
$$

Let $I_{n k}$ denote the interval with end points $k / 2^{n},(k+2) / 2^{n}$, where $k$ and $n$ are arbitrary integers. Denote by $Q_{\gamma v}$ the set of all points of $P_{\gamma}^{\nu}$ contained in $I_{n k}$. If $Q_{00}$ is not a null class, there is an index $g$ such that $Q_{00}$ is not null, while $Q_{g+1,0}$ is a null class; furthermore there is either an index $h$ such that $Q_{g h}$ is not null, while $Q_{g, h+1}$ is a null class, or else $Q_{g \Omega}$ exists and we may set $h=\Omega$.

At a point $y=k / 2^{n}$ we set

$$
f_{n}(y)=m\left(f, Q_{g h}\right)
$$

where $m\left(f, Q_{g h}\right)$ denotes the minimum of $f(x)$ on $Q_{g h}$. If $I_{n k}$ contains no point of $P=P_{0}$, that is if $Q_{00}$ is a null class, we set $f_{n}(y)=0$. In each interval $\left(k / 2^{n},(k+1) / 2^{n}\right), f_{n}(y)$ is to be linear, its values in the interval being determined by the values at the end points.

For a given point $x$ of $P$ we consider two cases: $1^{\circ}$ the point $x$ belongs to a set $P_{\gamma}^{\nu}-P_{\gamma}^{\nu+1} ; 2^{\circ}$ the point $x$ belongs to a set $P_{\gamma}^{\Omega}-P_{\gamma+1}$. In the first case $x$ is an isolated point of $P_{\gamma}^{\nu}$. Hence for sufficiently large $n$, any interval $I_{n k}$ containing $x$ will determine a class $Q_{g h}=Q_{y \nu}$ consisting of the single point $x$, and we will have

$$
f_{n}(x)=f(x) .
$$

In the second case, for $n$ sufficiently large, no point of $P_{\gamma+1}$ will lie in any interval $I_{n k}$ containing $x$. Since in this case $f(x)$ is continuous at $x$ relative to $P_{\gamma}^{\Omega}$ there is an interval $I_{n_{1} k_{1}}$ containing $x$ on which $\omega\left(f, Q_{\gamma \Omega}\right)<e$, where $e$ is any small positive number and $n_{1}$ is dependent on $e$, and $Q_{\gamma \Omega}$ is the portion of $P_{\gamma}^{\Omega}$ contained in $I_{n_{1} k_{1}}$. It follows that

\footnotetext{
${ }^{*}$ Cf. Baire, loc. cit., $\$ \S 57,58$.
} 


$$
\left|f(x)-m\left(f, Q_{\gamma \Omega}\right)\right|<e .
$$

On any interval $I_{n k}$ containing $x$ and interior to $I_{n_{1} k_{1}}, f_{n}(x)$ lies between $M\left(f, Q_{\gamma \Omega}\right)$ and $m\left(f, Q_{\gamma \Omega}\right)$. Hence for every $n \geqq n$, we have

$$
\left|f(x)-f_{n}(x)\right| \geqq e .
$$

The sequences $\left\{f_{n}(x)\right\}$ converge to $f(x)$ on $P$. The functions $f_{n}(x)$ are continuous on $I$ and therefore on $P$. We shall show that the convergence is relatively uniform by establishing relatively uniform convergence on each of the enumerable system of sets $P_{\gamma}-P_{\gamma+1}$ * $^{*}$

This set has the representation

$$
P_{\gamma}-P_{\gamma+1}=\sum_{\nu} P_{\gamma}^{\nu}-P_{\gamma}^{\nu+1}+P_{\gamma}^{\Omega}-P_{\gamma+1} .
$$

The set $P_{\gamma}^{\nu}-P^{\nu+1}$ is enumerable. Hence $\sum_{\nu} P_{\gamma}^{\nu}-P_{\gamma}^{\nu+1}$ is enumerable and the convergence is relatively uniform on this part of $P_{\gamma}-P_{\gamma+1}$.

The set $P_{\gamma}^{\Omega}$ is perfect, and $P_{\gamma+1}$ is closed and non-dense on $P_{\gamma}^{\Omega}$. Therefore $P_{\gamma}^{\Omega}-P_{\gamma+1}$ consists of an enumerable infinity of portions of $P_{\gamma}^{\Omega}$, except for the end points which belong to $P_{\gamma+1}$. Let $\left(a_{1}, b_{1}\right)$ denote a portion of $P_{\gamma}^{\Omega}$ with end points $a_{1}, b_{1}$, and let $(\zeta, \eta)$ denote a portion interior to $\left(a_{1}, b_{1}\right)$. On the set $(\zeta, \eta), f(x)$ is continuous. It follows readily from the definition of $f_{n}(x)$ that the sequence $\left\{f_{n}(x)\right\}$ converges uniformly on $(\zeta, \eta)$. Since the portion $\left(a_{1}, b_{1}\right)$ of $P_{\gamma}^{\Omega}-P_{\gamma+1}$ is the sum of an enumerable set of portions $(\zeta, \eta)$ it follows that the convergence is relatively uniform on $\left(a_{1}, b_{1}\right)$. For the same reason it is relatively uniform on $P_{\gamma}^{\Omega}-P_{\gamma+1}$ (and therefore on $P$ as was to be proved).

From theorems 1 and 2 we have:

Theorem 3. A necessary and sufficient condition that a function $f(x)$ defined on an interval $I$ be the limit of a sequence of continuous functions converging relatively uniformly on $I$ is that the discontinuities of $f(x)$ relative to every perfect subset $P$ of $I$ be non-dense on $P$.

This theorem can be extended following the methods of Baire to spaces of $n$ or zero dimensions. $\dagger$

4. We shall now give a definition of a function $f(x)$ which is everywhere represented by its Fourier Series, although the Fourier Series is not relatively uniformly convergent in any interval.

Let $E$ denote any enumerable dense subset of the interval $I \equiv(0 \leqq x \leqq 2 \pi)$ and let

$$
x_{1}, x_{2}, x_{3}, x_{4} \cdots x_{n}, \cdots
$$

be the elements of $E$ in some order. Then

* Cf. the previous paper, Propositions VII. and VIII.

† Cf. loc. cit. and A c t a M a the mati c a, vol. 32 (1909), p. 97-175. 


$$
f(x)=\sum_{n=1}^{\infty} \frac{1}{2^{n}} \operatorname{sgn}\left(x-x_{n}\right) * *
$$

The series (1) converges uniformly. Hence for every small positive number $e$ there is an integer $n_{e}$ such that for all $n \geqq n_{e}$

$$
\left|\sum_{n}^{\infty} \frac{1}{2^{k}} \operatorname{sgn}\left(x-x_{k}\right)\right| \leqq e / 2
$$

uniformly in $x$.

The function $f(x)$ is continuous at every point $x$ not in $E$. Choose $n_{e}$ effective in inequality (2). Of the elements $x_{n}\left(n \leqq n_{e}\right)$ there is one $x_{\bar{n}}$ which is nearest to $x$. Suppose $x^{\prime}$ such that $\left|x-x^{\prime}\right|<\left|x-x_{\bar{n}}\right|$. Then for every $n \leqq n_{e}$,

$$
\operatorname{sgn}\left(x-x_{n}\right)=\operatorname{sgn}\left(x^{\prime}-x_{n}\right) .
$$

From the preceding considerations

$\left|f(x)-\sum_{n=1}^{n=n_{e}} \frac{1}{2^{n}} \operatorname{sgn}\left(x-x_{n}\right)\right| \leqq e / 2, \quad\left|f\left(x^{\prime}\right)-\sum_{n=1}^{n=n_{e}} \frac{1}{2^{n}} \operatorname{sgn}\left(x^{\prime}-x_{n}\right)\right| \leqq e / 2$,

and therefore, applying equation (3),

$$
\left|f(x)-f\left(x^{\prime}\right)\right|<e
$$

for every $x^{\prime}$ sufficiently near $x$.

In case $x=x_{j}$ we choose $n_{e}>j$ effective in inequality (2), obtaining

$$
\left|f(x)-\frac{\operatorname{sgn}\left(x-x_{j}\right)}{2^{j}}-\sum_{n=1}^{n_{e}} \frac{1}{2^{n}} \operatorname{sgn}\left(x-x_{n}\right)\right| \leqq e .
$$

It is easy to see from the previous discussion that

$$
f(x)-\frac{1}{2^{j}} \operatorname{sgn}\left(x-x_{j}\right)
$$

is continuous at $x_{j}$. Hence

Consequently

$$
f\left(x_{j} \pm 0\right)=\sum_{n=1}^{n=\infty} \frac{\operatorname{sgn}\left(x_{j}-x_{n}\right)}{2^{n}} \pm \frac{1}{2^{j}} .
$$

$$
f\left(x_{j}+0\right)+f\left(x_{j}-0\right)=2 f\left(x_{j}\right),
$$

that is, $f(x)$ is a regular function, discontinuous only at the points of an enumerable dense set. We proceed to determine the total variation of $f(x)$. Let $\mu(x)=0(x \leqq 0) ; \mu(x)=1(x>0)$. Then $\phi(x)=\sum_{u=1}^{n=\infty} 2^{-n} \mu\left(x-x_{n}\right)$ is an increasing function of $x$ and $\phi(2 \pi)=1$. Since

$$
\operatorname{sgn}(x)=\mu(x)-\mu(-x)
$$

${ }^{*} \operatorname{Sgn} x$ is the Kronecker function equal to $-1,0,+1$ when $x$ is negative, zero, positive, respectively. 
we have $\phi(x)=\phi(x)-\phi(-x)$, and hence the total variation of $f(x)$ in the interval $I$ is 2 . It follows from a well known theorem of Lebesgue that $f(x)$ is everywhere represented by its Fourier Series.* Since the discontinuities of $f(x)$ are dense on $I$ the series is not relatively uniformly convergent.

5. Denote by $R_{1}$ the class of all functions which are limits of sequences of continuous functions converging relatively uniformly, and by $R_{2}$ the corresponding extension of $R_{1}$, etc. We thus obtain a classification of functions in terms of relatively uniform convergence. The following questions are at once suggested. What is the relation of this classification to the Baire classification? Are there functions in the Baire series of classes which do not come under this new classification, that is, does there exist a number $\alpha$ such that $R_{a}=R_{a+1}$ ? The answers to these questions are not yet in my possession. The following facts are however of interest in this connection.

Baire shows that every function of class 1 is a limit of a uniformly convergent sequence of functions $F_{a}$, which functions are in fact of class $R_{1}$. Hence the class $R_{2}$ contains class 1 of Baire. It also contains functions of class 2 , as the following example shows. Let $f_{n}(x)=0$ if $x$ is not equal to $p / 2^{m}(m \leqq n)$, and $f_{n}(x)=1$ if $x=p / 2^{m}(m \leqq n)$. Then $f(x)=L_{n} f_{n}(x)=1$ if $x=p / 2^{m}$, $f(x)=0$ if $x \neq p / 2^{m}$ ( $m$ and $p$ integers). The sequence $\left\{f_{n}(x)\right\}$ converges uniformly except on the enumerable set of values $\left\{p / 2^{m}\right\}$. Hence the convergence is relatively uniform. The function $f(x)$ is totally discontinuous, and therefore does not belong to the Baire class 1 .

The further discussion of this subject involves a detailed analysis of the Baire classes 2 and 3 which is not yet complete, and which furthermore lies beyond the scope of the present paper.

* Cf. Leçons sur les séries trigonométriques, p. 73.

UNIVERSITY OF Iowa. 